Neth. J. Agric. Sci., 17 (1969) : 71-79

\title{
Measuring thermal conductivity of soils under laboratory conditions
}

\author{
A. I. Golovanov \\ Moscow Hydromeliorative Institute, Moscow, USSR \\ Received 29 October, 1968
}

\section{Summary}

A series of experiments concerning the determination of the thermal conductivity of soils has been described. When a thin needle used as heating element is placed along the main axis of a cylindrical sample, the thermal conductivity can be computed fairly accurately from a simplified equation (3) when the conditions as given in Fig. 1 are satisfied. For measurements during a period of 100 seconds the diameter of the sample must be at least $4 \mathrm{~cm}$.

If heating elements are placed horizontally, for instance to measure simultaneously the thermal conductivity of different soil layers, it was found that their distance must be at least $10 \mathrm{~cm}$. Concerning the convection of heat it was found that the measurements should preferably not exceed 100 seconds. Finally it was investigated to what extent thermal conductivity measurement can be used to determine flow velocities of water in soils. For coarse sand the method is able to detect real flow velocities higher than some $0.35 \mathrm{~cm} / \mathrm{min}$.

\section{Introduction}

The thermal conductivity is an important factor in the heat regime of soils and needs to be known for various types of calculations of the heat balance of the earth's surface (Chudnovsky, 1954; Van Duin, 1956; Van Wijk, 1963). Since its magnitude largely depends on the moisture content of the soil it is also used as an indirect method for the determination of the moisture content. Examples of the latter use are given by Van Duin and De Vries (1954), and Youngs (1956). When determining the moisture content of the soil by measuring the thermal conductivity it is important to know the effect of the duration of the measurement, the size of the sample and the amount of energy to be applied. In an experimental setup to measure the flow of water in unsaturated soils, the moisture determination by means of heat conductivity was chosen by the Division of Hydrology of the Institute for Land and Water Management Research at Wageningen, The Netherlands ${ }^{1}$. In order to get some insight in the effect of the above mentioned factors, a series of experiments was carried out. The results are described in this article.

1 The author spent in 1968 a 9 -month period as visiting-scientist at that Institute. 


\section{Measuring techniques}

One of the methods for the measurement of the thermal conductivity of a soil is based on observing the rise in temperature of an electrically heated cylindrical element (needle) stuck into the soil. For a thin and infinitely long element the temperature field in an infinite medium can be described by the equation

$\vartheta-\vartheta_{0}=-\frac{\mathrm{q}}{4 \pi \lambda} \mathrm{E}_{\mathrm{i}}\left(-\frac{\mathrm{r}^{2}}{4 x \mathrm{t}}\right)$

where $\vartheta=$ temperature in any arbitrary point at a distance $\mathrm{r}$ from the centre of needle in ${ }^{\circ} \mathrm{C}$;

$\mathrm{t}=$ time in sec.;

$\eta_{0}=$ initial $(\mathrm{t}=0)$ temperature in ${ }^{\circ} \mathrm{C}$;

$\mathrm{q}=$ steady input of heat per unit length of needle in $\mathrm{cal} / \mathrm{sec} . \mathrm{cm}$;

$\lambda=$ thermal conductivity in $\mathrm{cal} / \mathrm{cm}$. $\mathrm{sec} .{ }^{\circ} \mathrm{C}$;

$x=$ thermal diffusivity defined as $x=\frac{\lambda}{\varrho \mathrm{c}}$ in $\mathrm{cm}^{2} / \mathrm{sec}$;

$\varrho=$ density of the soil in $\mathrm{g} / \mathrm{cm}^{3}$;

$\mathrm{c}=$ specific heat in $\mathrm{cal} / \mathrm{g} .{ }^{\circ} \mathrm{C}$.

For $\mathbf{t}>\mathrm{r}^{2 / 4} \pi$ it is possible to simplify equation (1) as

$\vartheta-\vartheta_{0}=\frac{\mathrm{q}}{4 \pi \lambda}\left(\ln \frac{4 x}{\mathrm{r}^{2}} \mathrm{t}-0.5772\right)$

If the rise of the temperature $\Delta \vartheta$ during time $\Delta t=t_{2}-t_{1}$ is known one can calculate the thermal conductivity from the equation

$\lambda=\frac{\mathrm{q}}{4 \pi \Delta \vartheta} \ln \mathrm{t}_{2} / \mathrm{t}_{1}$

There are several publications in which this method and the apparatuses used have been given (for example De Vries, 1952; Van Drunen, 1949; Janse and Borel, 1965).

\section{Size of soil samples}

Under laboratory conditions one deals with soil samples of finite size. The problem is then for what size of samples the simplified equation (3) is valid. It is clear that a small sample kept at a constant temperature at its surfaces will show a smaller rise of temperature inside the sample than an infinite sample. If $\lambda$ is then calculated by equation (3) the value found will be too high. On the other hand, if the surfaces of the sample are perfectly insulated this will result in a $\lambda$ too low. Between these two extremes are cases with a certain heat flux through the surfaces, giving smaller errors.

The magnitude of the error introduced by the size of the sample also depends on the duration of the measurement and on the heat properties of the soil. As far as these factors are concerned the error can be estimated theoretically. To this purpose two theoretical solutions can be applied that originally were derived for groundwater 
flow (see Muskat, 1946). The first one can (after an appropriate conversion) describe the temperature $\eta^{*}$ in an infinitely long cylinder which has in its centre a thin linear heat source and a constant temperature $\eta_{0}$ at its surfaces.

$\vartheta^{\prime}=2 \ln \frac{\mathrm{R}}{\mathrm{r}}-4 \sum_{\mathrm{n}=1}^{\infty} \frac{\mathrm{J}_{0}\left(\zeta \mu_{\mathrm{n}}\right)}{\mu_{\mathrm{n}}^{2} J_{1}^{2}\left(\mu_{\mathrm{n}}\right)} \mathrm{e}^{-\mu_{\mathrm{n}}^{2} \chi / \mathrm{R}^{2}}$

where $\vartheta^{\prime}=\frac{4 \pi \lambda}{\mathrm{q}}\left(y^{*}-\vartheta_{0}\right)$ relative temperature rise;

$\mathrm{R}=$ radius of the cylinder in $\mathrm{cm}$;

$\zeta=\mathrm{r} / \mathrm{R}$

$\mathrm{r}=$ the distance of any arbitrary point from the axis of the cylinder in $\mathrm{cm}$;

$\chi=\varkappa \mathrm{t}$ in $\mathrm{cm}^{2}$;

$\mu_{\mathrm{n}}=$ roots of the function $\mathrm{J}_{0}\left(\mu_{\mathrm{n}}\right)=0$.

A second solution pertains to the temperature $\vartheta^{* * *}$ of an infinitely long cylindrical sample with insulated surfaces. This solution is:

$\eta^{\prime \prime}=2 \ln \mathrm{R} / \mathrm{r}+\zeta^{2}+\frac{4 \chi}{\mathrm{R}^{2}}-3 / 2-4 \sum_{\mathrm{n}=1}^{\mathrm{n}=\infty} \frac{\mathrm{J}_{0}\left(\zeta \mu_{\mathrm{n}}\right)}{\mu_{\mathrm{n}}^{2} \mathrm{~J}_{0}^{2}\left(\mu_{\mathrm{n}}\right)} \mathrm{e}^{-\mu_{\mathrm{n}}^{2} \chi / \mathrm{R}^{2}}$

where $\eta^{\prime \prime}=\frac{4 \pi \lambda}{\mathrm{q}}\left(\eta^{* *}-y_{0}\right)$ relative temperature rise;

$$
\mu_{\mathrm{n}}=\text { roots of the function } J_{1}\left(\mu_{\mathrm{n}}\right)=0 \text {. }
$$

A digital computer program was developed to calculate the relative temperature rise for sample radii of $\mathrm{R}=1,2,3,5,7,10 \mathrm{~cm}$ and for a $0.05 \mathrm{~cm}$ radius of the needles. The values obtained were compared with the results of equation (1), for which the value $\mathbf{R}=\infty$ was assumed (Table 1 ).

Using these results it was found that the radius $R$ of the samples for which equation (3) can be used for determining $\lambda$ with an error $|\Delta|<1 \%$, can be represented by the region below the curve in Fig. 1. If measurements are carried out during a period $\mathrm{t}=100 \mathrm{sec}$ and $x=(3$ to 6$) \cdot 10^{-3} \mathrm{~cm}^{2} / \mathrm{sec}$ the radius of the sample must be $\mathrm{R} \geqslant 1.1$ to $1.4 \mathrm{~cm}$. For a measuring time $\mathrm{t}=300 \mathrm{sec}$ one finds $\mathrm{R} \geqslant 1.8$ to $2.5 \mathrm{~cm}$ accordingly.

To check these calculations some laboratory experiments have been carried out. The apparatus and techniques used were the same as those described by Janse and Borel (1965). The increase in temperature was taken by a recording millivoltmeter type Servogor. Very fine sand ('Blokzijl' sand) was used, having a dry bulk dentity of $1.16 \mathrm{~g} / \mathrm{cm}^{3}$ and a moisture content of $19.4 \%$ by volume. Temperature measurements were carried out in copper cylinders $\mathrm{R}=1.0,2.1,3.0,5.0,7.0$ and $10.0 \mathrm{~cm}$ diameter and a length of $10 \mathrm{~cm}$. The length of the needles was $8.5 \mathrm{~cm}$. Top and bottom of cylinders were carefully insulated by a sheet of poly-urethane foam to obtain an exact radial flow of heat. In one series of measurements the walls of the cylinders were kept at a constant temperature by placing them in a water-thermostat chamber restricting temperature fluctuations to $\pm 0.01{ }^{\circ} \mathrm{C}$. In another series the walls of cylinders were insulated by a glass-wool blanket. The results of these measurements 
Table 1 Relative temperature rise according eq. (1), $R=\infty$; eq. (4), 9 '; and eq. (5), $9 \prime \prime$

\begin{tabular}{|c|c|c|c|c|c|c|c|c|c|c|c|c|c|c|}
\hline \multirow{2}{*}{$\begin{array}{l}\chi \\
c m^{2}\end{array}$} & \multicolumn{2}{|c|}{$R=1 \mathrm{~cm}$} & \multicolumn{2}{|c|}{$R=2 \mathrm{~cm}$} & \multicolumn{2}{|c|}{$R=3 \mathrm{~cm}$} & \multicolumn{2}{|c|}{$R=5 \mathrm{~cm}$} & \multicolumn{2}{|c|}{$R=7 \mathrm{~cm}$} & \multicolumn{2}{|c|}{$R=10 \mathrm{~cm}$} & \multicolumn{2}{|c|}{$R=\infty$} \\
\hline & $\vartheta^{\prime}$ & $\eta)^{\prime \prime}$ & $\vartheta^{\prime}$ & $9^{\prime \prime}$ & $y^{\prime}$ & $99^{\prime \prime}$ & $\vartheta^{\prime}$ & $9^{\prime \prime}$ & $\varphi^{\prime}$ & $\%^{\prime \prime}$ & $\vartheta^{\prime}$ & $\eta^{\prime \prime}$ & $\left(\vartheta-\vartheta_{0}\right)$ & $4 \pi d$ \\
\hline 0 & 0 & 0 & 0 & 0 & 0 & 0 & 0 & 0 & 0 & 0 & 0 & 0 & 0 & \\
\hline 0.03 & 3.315 & 3.315 & 3.315 & 3.315 & 3.315 & 3.315 & 3.315 & 3.315 & 3.315 & 3.315 & 3.315 & 3.315 & 3.315 & \\
\hline 0.06 & 3.998 & 3.998 & 3.998 & 3.998 & 3.998 & 3.998 & 3.998 & 3.998 & 3.998 & 3.998 & 3.998 & 3.998 & 3.998 & \\
\hline 0.09 & 4.400 & 4.400 & 4.400 & 4.400 & 4.400 & 4.400 & 4.400 & 4.400 & 4.400 & 4.400 & 4.400 & 4.400 & 4.400 & \\
\hline 0.12 & 4.685 & 4.686 & 4.685 & 4.685 & 4.685 & 4.685 & 4.685 & 4.685 & 4.685 & 4.685 & 4.685 & 4.685 & 4.685 & \\
\hline 0.15 & 4.906 & 4.909 & 4.908 & 4.908 & 4.908 & 4.908 & 4.908 & 4.908 & 4.908 & 4.908 & 4.908 & 4.908 & 4.908 & \\
\hline 0.18 & 5.084 & 5.095 & 5.089 & 5.089 & 5.089 & 5.089 & 5.089 & 5.089 & 5.089 & 5.089 & 5.089 & 5.089 & 5.089 & \\
\hline 0.24 & 5.353 & 5.405 & 5.376 & 5.376 & 5.376 & 5.376 & 5.376 & 5.376 & 5.376 & 5.376 & 5.376 & 5.376 & 5.376 & \\
\hline 0.30 & 5.540 & 5.674 & 5.599 & 5.599 & 5.599 & 5.599 & 5.599 & 5.599 & 5.599 & 5.599 & 5.599 & 5.599 & 5.599 & \\
\hline 0.45 & 5.802 & 6.292 & 6.003 & 6.004 & 6.003 & 6.003 & 6.003 & 6.003 & 6.003 & 6.003 & 6.003 & 6.003 & 6.003 & \\
\hline 0.60 & 5.912 & 6.894 & 6.289 & 6.293 & 6.291 & 6.291 & 6.291 & 6.291 & 6.291 & 6.291 & 6.291 & 6.291 & 6.291 & \\
\hline 0.90 & 5.997 & 8.094 & 6.679 & 6.717 & 6.696 & 6.696 & 6.696 & 6.696 & 6.696 & 6.696 & 6.696 & 6.696 & 6.696 & \\
\hline 1.20 & 5.989 & 9.294 & 6.925 & 7.058 & 6.983 & 6.984 & 6.983 & 6.983 & 6.983 & 6.983 & 6.983 & 6.983 & 6.983 & \\
\hline 1.50 & 5.991 & 10.494 & 7.085 & 7.372 & 7.203 & 7.210 & 7.206 & 7.206 & 7.206 & 7.206 & 7.206 & 7.206 & 7.206 & \\
\hline 1.80 & 5.991 & 11.694 & 7.188 & 7.676 & 7.379 & 7.400 & 7.389 & 7.389 & 7.389 & 7.389 & 7.389 & 7.389 & 7.389 & \\
\hline 2.40 & 5.991 & 14.094 & 7.298 & 8.278 & 7.640 & 7.722 & 7.676 & 7.676 & 7.676 & 7.676 & 7.676 & 7.676 & 7.676 & \\
\hline 3.00 & 5.991 & 16.494 & 7.344 & 8.878 & 7.815 & 8.010 & 7.899 & 7.900 & 7.899 & 7.899 & 7.899 & 7.899 & 7.899 & \\
\hline 4.50 & 5.991 & 22.494 & 7.374 & 10.378 & 8.046 & 8.688 & 8.300 & 8.311 & 8.305 & 8.305 & 8.305 & 8.305 & 8.305 & \\
\hline 6.00 & 5.991 & 28.494 & 7.377 & 11.878 & 8.134 & 9.356 & 8.569 & 8.621 & 8.592 & 8.593 & 8.592 & 8.592 & 8.592 & \\
\hline
\end{tabular}

are shown in Fig. 2. In this figure the relation between $\lambda_{R}$ and $\lambda_{\infty}$ is given. The value of $\lambda_{R}$ is that computed by equation (3) from measurements in cylinders with radius $\mathrm{R}$ respectively $1,2,3,5$ and $7 \mathrm{~cm}$, and $\lambda_{\infty}$ is that computed from the measurements in the cylinders with radius $R=10 \mathrm{~cm}$. The values of $\lambda$ were computed for various time intervals namely for $\mathrm{t}=10$ to $100 \mathrm{sec}$, for $\mathrm{t}=100$ to $300 \mathrm{sec}$ and for $\mathrm{t}=$ 300 to $1000 \mathrm{sec}$. Comparison of these data with the calculations by equations (1), (4) and (5) (with $\lambda=2.2 \cdot 10^{-3} \mathrm{cal} / \mathrm{cm}$. sec. ${ }^{\circ} \mathrm{C} ; ~ \varkappa=4.87 \cdot 10^{-3} \mathrm{~cm}^{2} / \mathrm{sec}$ ) confirmed our theoretical conclusion about influence of the size of soil samples.

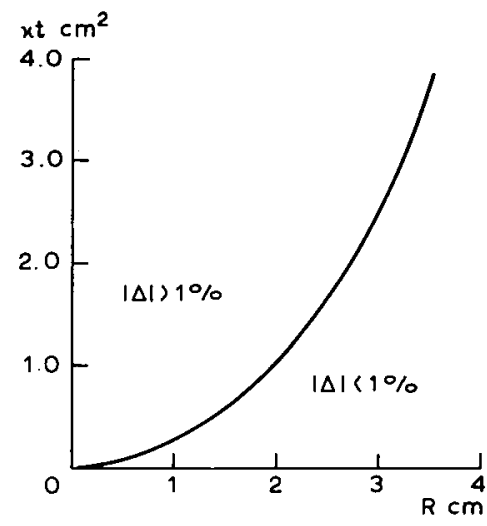

Fig. 1 Minimum radius of cylindrical soil samples in which the thermal conductivity $\lambda$ can be determined by eq. (3) with an error $|\triangle|<1 \%$ 

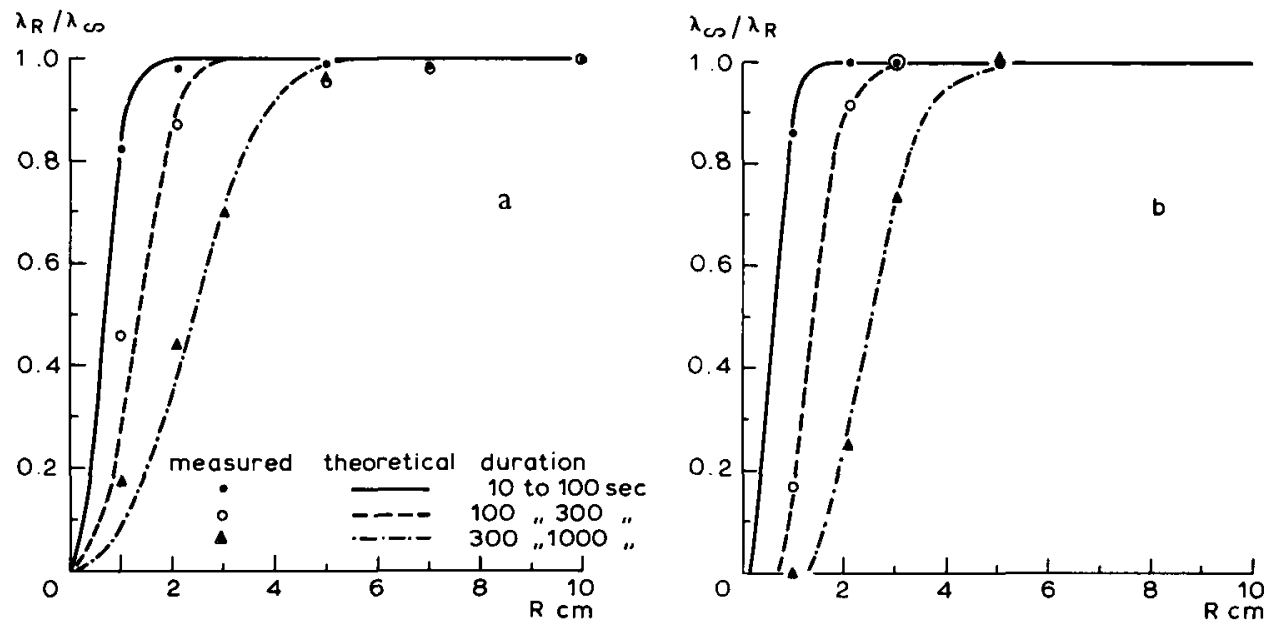

Fig. 2 Influence of the radius of the sample $R$ on the determination by means of eq. (3) of the thermal conductivity of a soil. a: samples with insulated walls; $b:$ samples with the walls at $a$ constant temperature

Sometimes measurements of thermal conductivity have to be carried out in cylindrical samples longer than the length of the needle, for instance if one wants to know heat properties of the soil at various depths. In these cases the needle is to be inserted perpendicular to the main axis of the cylinder and in that case one deals with a rather complicated three-dimensional flow of heat, for which no theoretical solutions are available. In order to check in how far equation (3) may be used for the determination of thermal conductivity under such conditions, a series of measurements were done in cylinders with a radius of $6 \mathrm{~cm}$ and of $10 \mathrm{~cm}$ length. The 'Blokzijl' sand in these experiments had a dry bulk density of $1.38 \mathrm{~g} / \mathrm{cm}^{3}$ and moisture contents of respectively $11.6 \%$ and $35.6 \%$ by volume. Comparison of values of $\lambda$ taken from these experiments with those computed from measurements with the needle through the main axis of the cylinders did show, that during $100 \mathrm{sec}$ observations the difference between the two values was not larger than $2 \%$. For example in sand with a moisture content of $11.6 \%, 15$ observations obtained from measurements with the needle through the main axis of the sample gave a mean value of $\lambda=2.338 .10^{-3}$ $\mathrm{cal} / \mathrm{cm}$. sec. ${ }^{\circ} \mathrm{C}$ with a standard deviation $\sigma=0.021 \cdot 10^{-3} \mathrm{cal} / \mathrm{cm}$. sec. ${ }^{\circ} \mathrm{C}$. A series of 12 observations with the heating needle perpendicular to the main axis of the sample gave a mean value of $\lambda=2.435 \cdot 10^{-3}$ with $\sigma=0.011 .10^{-3}$. A statistical analysis gave as a result $\triangle \lambda=0.047$ with a standard deviation $\sigma_{\triangle}=0.024$, so no significant difference between the results of the two ways of measuring was found.

When the needle is inserted perpendicular to the main axis, it is also of interest to know the influence of the length of the sample on $\lambda$ as determined by means of equation (3). For this purpose cylinders were used with a radius of $6 \mathrm{~cm}$ and length of respectively $5,7,10,15$ and $20 \mathrm{~cm}$. The surface of the samples were carefully insulated. In Table 2 mean values from 6 repetitions of $\lambda$ are given. The data in Table 2 show, that in the case of measurements with some horizontally inserted needles in a soil column, the vertical distance between the elements must for practical 
Table 2 Influence of the length of cylindrical samples of a sandy soil on the determination of the thermal conductivity according eq. (3), the heating needle inserted perpendicular to the main axis

\begin{tabular}{|c|c|c|c|c|}
\hline \multirow{2}{*}{$\begin{array}{l}\text { Length of } \\
\text { cylinder } \\
(\mathrm{cm})\end{array}$} & \multicolumn{2}{|c|}{ Moisture content $11.6 \%$ by vol. } & \multicolumn{2}{|c|}{ Moisture content $35.6 \%$ by vol } \\
\hline & $\lambda \cdot 103$ & $\lambda_{1} / \lambda_{10}$ & $\lambda \cdot 103$ & $\lambda_{1} / \lambda_{10}$ \\
\hline 5 & 2.25 & 0.926 & 3.13 & 0.954 \\
\hline 7 & 2.36 & 0.971 & 3.19 & 0.972 \\
\hline 10 & 2.43 & 1.000 & 3.28 & 1.000 \\
\hline 15 & 2.43 & 1.000 & - & - \\
\hline 20 & 2.46 & 1.012 & - & - \\
\hline
\end{tabular}

purposes be $10 \mathrm{~cm}$ or more in order to prevent mutual influences. This conclusion holds only if equation (3) is used for the calculation of $\lambda$, and the duration of measuring is $100 \mathrm{sec}$.

\section{Influence of convection on heat transport}

If some part of a porous medium containing water is heated, there will be a supplementary heat transport due to the convection flow of the water. The effect of this phenomenon depends on the duration of heating and on the moisture content of the porous medium. To get an insight in this effect some measurements have been carried out with 'Blokzijl' sand, having a dry bulk density of $1.3 \mathrm{~g} / \mathrm{cm}^{3}$ and moisture contents of respectively $0.3,13.9,26.2$ and $36.3 \%$ by volume. Samples were prepared in cylinders with a length of $10 \mathrm{~cm}$ and a radius of $10 \mathrm{~cm}$. The ends of the samples were insulated. For heating, currents of $\mathrm{I}=0.036$ to $0.800 \mathrm{amp}$. were applied. The temperature rise after $100 \mathrm{sec}$. was $\Delta \vartheta=0.35$ to $52.0^{\circ} \mathrm{C}$ respectively and after $300 \mathrm{sec} . \triangle \vartheta=0.39$ to $58.0^{\circ} \mathrm{C}$. In Fig. 3 the relation between $\lambda$ and the current is shown. The values of $\lambda$ are those determined by observations of temperature rise during 10 to $100 \mathrm{sec}$. Scatter values of $\lambda$ from its mean show that there is practically no dependency of $\lambda$ on I. This means, that the influence of convection on heat transport during this time is negligeable. In almost all experiments, however, a relatively smaller rises in temperature occurred from 100 to 300 seconds than in the period from 10 to 100 seconds. This implies an increasing $\lambda$ and hence an increasing heat transport in the first mentioned period. This is shown in Fig. 4 in which the ratio $\lambda_{300} / \lambda_{100}$ is given for different currents and different moisture contents. The lines in this figure, drawn by the method of the least squares, are sloping into the direction of an increasing current. This increase of $\lambda$ is proportional with the moisture content of the soil and it is therefore reasonable to ascribe this to supplementary heat transport caused by a convection flow of the water. For very dry sand with a moisture content of $0.3 \%$ by volume it may be due to convection of water vapour and air. Fig. 4 does show a decreasing influence of convection at higher currents, that is at higher temperatures (at current of 0.5 to $0.8 \mathrm{amp}$. there is practically no increase of $\lambda$ ). This phenomenon can be explained by a decreasing of $\lambda$ due to dessication of the soil near the needle.

From the above it can be concluded that over short time intervals (near $100 \mathrm{sec}$.) should be used to determine the thermal conductivity of the soil. 


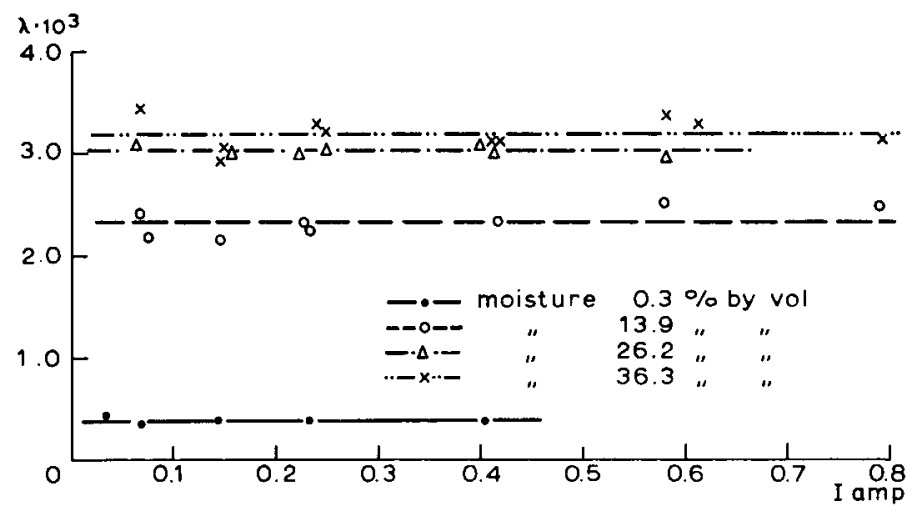

Fig. 3 Relation between thermal conductivity determined during 10 to 100 seconds and current I

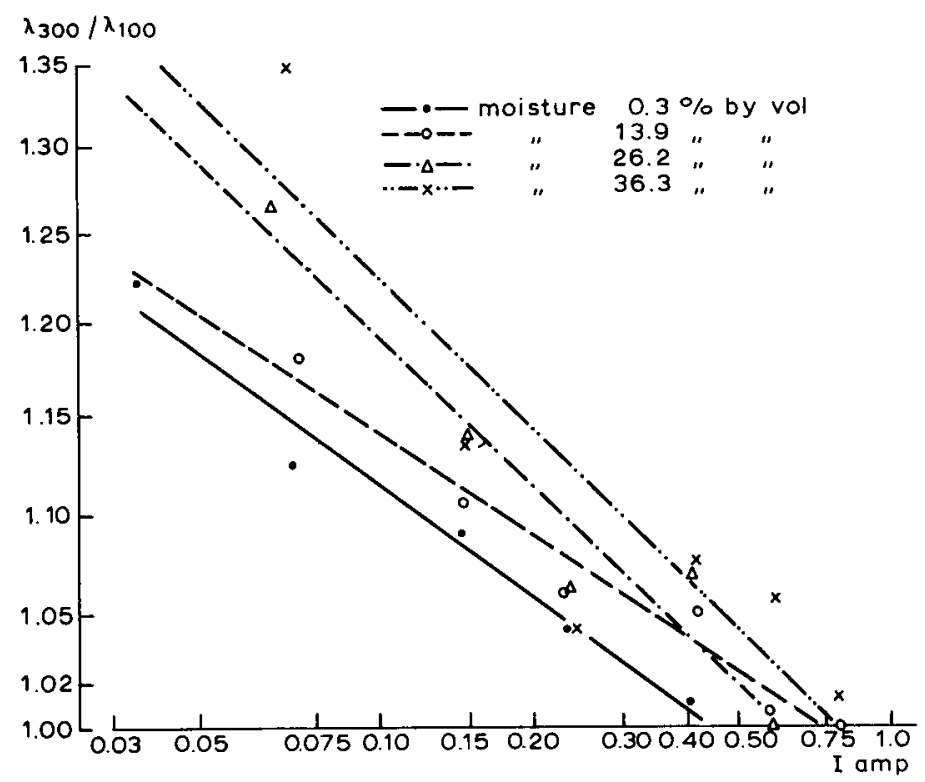

Fig. 4 Relative increase in the thermal conductivity due to convection

\section{Influence of water flow on heat transport}

Finally the influence of water moving through porous media on the determination of their thermal conductivity was considered. To this end the thermal conductivity was measured in a cylindrical column with a length of $40 \mathrm{~cm}$ and a radius of $6 \mathrm{~cm}$ filled with coarse sand (fraction 0.60 to $0.85 \mathrm{~mm}$, hydraulic conductivity 59 meters/ day) in which different flow velocities of water were created under saturated conditions. The results of the measurements are shown in Fig. 5. In this figure $\lambda_{\mathrm{v}}$ is the 


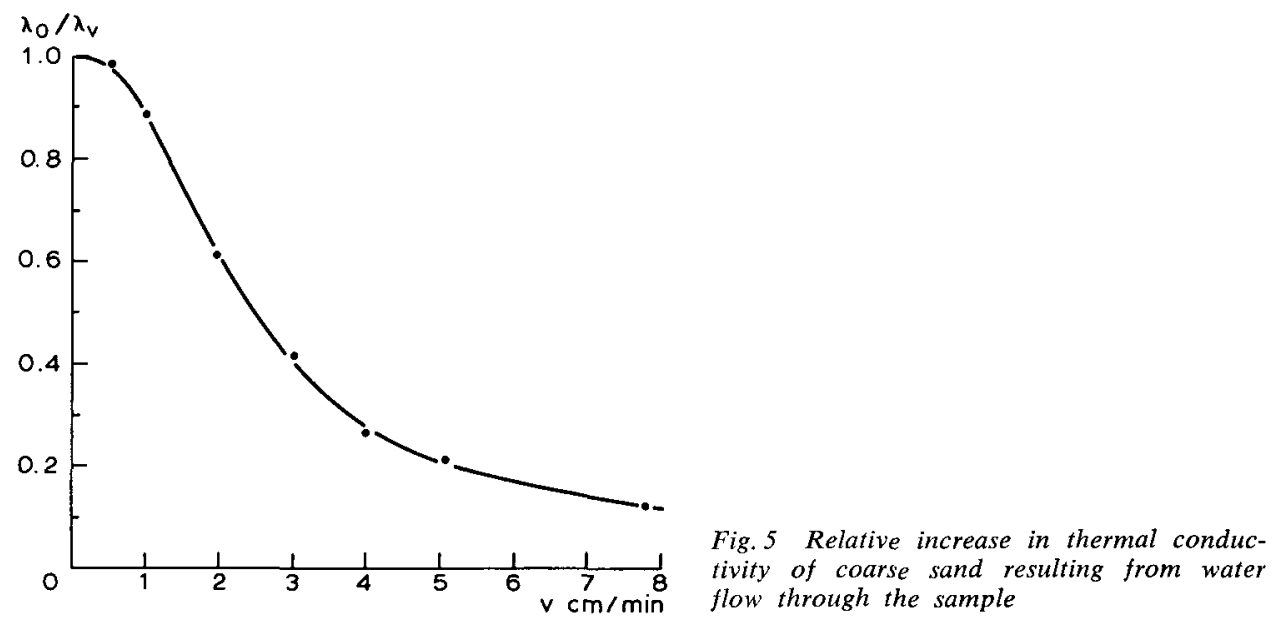

thermal conductivity of the medium, with water flowing through it at a velocity $\mathrm{v}$, $\lambda_{0}$ is the thermal conductivity with that velocity equal to zero. The flow velocity was defined as the mean real flow velocity in the sample measured at a temperature of $10^{\circ} \mathrm{C}$ and it was calculated by the formula

$\mathrm{v}=\frac{\mathrm{Q}}{\mathrm{Fp}}$

where $\mathrm{Q}=$ discharge of water through the column in $\mathrm{cm}^{3} / \mathrm{min}$;

$\mathrm{F}=$ cross-sectional area of the column in $\mathrm{cm}^{2}$;

$\mathbf{p}=$ porosity of the sand in $\%$.

Each of the points on Fig. 5 presents a mean of 4 measurements. In all the measurements an increase of $\lambda$ due to additional heat transport by the moving water could be observed. A noticeable increase (some 1.5 to $2 \%$ ) of $\lambda$ starts already when the flow velocity is $0.35 \mathrm{~cm} / \mathrm{min}$. The relation between $\lambda_{0} / \lambda_{\mathrm{v}}$ and $\mathrm{v}$ can be used, for example, to registrate the velocity of moving water through porous media, provided that the real flow velocity is larger than about $0.35 \mathrm{~cm} / \mathrm{min}$.

\section{Acknowledgement}

The author is greatly indebted to Dr. J. Wesseling and Mr. K. E. Wit of the Division of Hydrology, and Mr. W. van Doorne of the Section Mathematics for their aid and advice throughout his work at the Institute for Land and Water Management Research at Wageningen, The Netherlands.

\section{References}

Chudnovsky, A. F., 1954. Teploobmen y dispersnykh sredakh (Heat transfer in despersed media). Gostekhizdat. Moscow. 
Drunen, F. C. van, 1949. Meting van de warmtegeleiding in vloeistoffen (Measuring thermal conductivity in liquids). Thesis, Utrecht.

Duin, R. H. A. van, 1956. Over de invloed van grondbewerking op het transport van warmte, lucht en water in de grond (On the influence of tillage on conduction of heat, diffusion of air and infiltration of water in soil). Thesis, Wageningen. Versl. Landbk. Onderz. 62.7. Pudoc, Wageningen.

Duin, R. H. A. van \& Vries, D. A. de, 1954. A recording apparatus for measuring thermal conductivity and some results obtained with it in soil. Neth. J. Agric. Sci, 2: 168-175.

Janse, A. R. P. \& Borel, G., 1965. Measurement of thermal conductivity in situ in mixed materials, e.g. soils. Neth. J. Agric. Sci., 13: 57-62.

Muskat, M., 1946. The flow of homogeneous fluids through porous media. Ann. Arbor. Michigan.

vires, D. A. de, 1952. Het warmtegeleidingsvermogen van grond (The thermal conductivity of soils). Meded. Landbouwhogeschool Wageningen, Netherlands 52. 1.

Wijk, W. R. van, 1963. Physics of plant environment. North-Holland Publishing Company, Amsterdam.

Youngs, E. G., 1956. A laboratory method of following moisture content changes. Rapp. VIe Congres Intern. Sci. du Sol, Paris: 89-93.

\section{REVIEWS}

633.2:631.559

J. P. van den Bergh : An analysis of yields of grasses in mixed and pure stands. Thesis, Agric. Univ., Wageningen, 1968. Also published as Agric. Res. Rept No. 714. Pudoc, Wageningen. 71 pp., 50 Figs, 13 Tables, with Dutch summary, price: Dfl. 8.35.

To describe competition species must be compared with a standard. By conventional standards, such as grammes dry matter, tiller number and sq. cm leaf area, two species and even one species cannot be compared in the course of the season. Therefore a standard without dimensions was needed. Dividing yield of a species (expressed in any unit) in mixed culture by that in monoculture gave a figure without dimensions, called relative yield. 